\title{
Modelo harmônico para a estimativa da temperatura do ar média mensal em diferentes locais do Estado do Rio Grande do Sul
}

\author{
Harmonic model for estimating the average monthly air temperature in different locations \\ of Rio Grande do Sul State, Brazil
}

\author{
Alberto Cargnelutti Filho ${ }^{I}$ Ronaldo Matzenauer ${ }^{\text {II }}$ Jaime Ricardo Tavares Maluf ${ }^{\text {II }}$ Bernadete Radin $^{\text {II }}$
}

\section{RESUMO}

\begin{abstract}
O objetivo deste trabalho foi ajustar o modelo harmônico para a estimativa da temperatura do ar média mensal em 37 locais do Estado do Rio Grande do Sul, com os dados do período de 1931 a 2000. Em cada local, os dados de 1931 a 1990 (60 anos) foram utilizados para estimar os coeficientes do modelo harmônico, e os dados de 1991 a 2000 (10 anos), para a validação do modelo. Foram testadas a normalidade e homogeneidade de variâncias, estimadas tendências e incorporadas aos modelos harmônicos construídos com base nas ondas anual e semestral. Coeficientes linear, angular, de correlação linear de Pearson e de determinação, raiz do quadrado médio do erro e índices de WILLMOTT (1981) e de CAMARGO \& SENTELHAS (1997) foram utilizados para validação dos modelos. $O$ coeficiente de determinação $\left(R^{2}\right)$ do modelo harmônico foi elevado em todos os locais $\left(R^{2} \geq 0,9867\right)$, conferindo confiabilidade nas previsões futuras. $O$ modelo harmônico gerado, para cada um dos 37 locais do Estado do Rio Grande do Sul, é adequado para estimar a temperatura do ar média mensal.
\end{abstract}

Palavras-chave: série temporal, modelagem, regressão periódica.

\section{ABSTRACT}

The objective of this research was to adjust the harmonic model for the estimation of average monthly air temperature, in 37 locations of Rio Grande do Sul State, Brazil, with data from the period 1931 to 2000. In each locality, data from 1931 to 1990 (60 years) was used to estimate the coefficients of the harmonic model and from 1991 to 2000 (10 years) to validate the model. It was tested the normality and homogeneity of variance among months, estimated trends and incorporated into the models harmonic based in waves annual and semiannual. The coefficients linear, angular, correlation and determination, the root mean square error and the index of WILLMOTT (1981) and CAMARGO \& SENTELHAS (1997) were used to validate the models. The coefficient of determination $\left(R^{2}\right)$ of the harmonic model, was high in all sites $\left(R^{2} \geq 0.9867\right)$, giving reliability for future forecasts. The harmonic model generated for each of the 37 location in the the Rio Grande do Sul State, Brazil, is adequate to estimate the average monthly air temperature.

Key words: time series, modeling, periodic regression.

\section{INTRODUÇÃO}

Séries temporais possibilitam estudar possíveis mudanças, ao longo do tempo, em uma determinada variável. Assim, o estudo de séries temporais é um importante instrumento para, por meio do comportamento passado, ajustar um modelo matemático para tendências e previsões futuras, principalmente no momento em que tantas especulações estão sendo feitas sobre mudanças climáticas, especialmente a temperatura do ar. Porém, esses modelos empíricos são limitados para serem realizadas previsões de tendências futuras e são restritos a determinação das disponibilidades climáticas regionais. Modelos físicos que consideram a interação atmosfera-superfície são usados para projeções de climas futuros.

'Departamento de Fitotecnia, Centro de Ciências Rurais (CCR), Universidade Federal de Santa Maria (UFSM), 97105-900, Santa

Maria, RS, Brasil. E-mail: cargnelutti@pq.cnpq.br. Autor para correspondência.

"Fundação Estadual de Pesquisa Agropecuária (FEPAGRO), Porto Alegre, RS, Brasil. 
Modelos empíricos para estimar a temperatura mínima média decendial (CARGNELUTTI FILHO et al., 2006) e as temperaturas máxima e média decendiais (CARGNELUTTI FILHO et al., 2008b), em função da altitude, latitude e longitude, foram estabelecidos e considerados adequados para qualquer local do Estado do Estado do Rio Grande do Sul. No entanto, a modelagem específica por local, por meio da análise harmônica, e a incorporação de possíveis tendências locais podem oferecer maior precisão nas estimativas.

Os procedimentos de Box e Jenkins (modelos ARIMA) consistem em explicar uma variável por meio de valores passados dela mesma. Há modelos autorregressivos (AR), médias móveis (MA) e combinação entre autorregressivos e médias móveis (ARMA e ARIMA) que podem ser aplicados em séries temporais (SARTORIS, 2003; MORETTIN \& TOLOI, 2006). Além destes, a análise harmônica, ou análise de Fourier ou análise de regressão periódica, se propõe a isolar as harmônicas (ou ondas), que, atuando em conjunto, reproduzem o comportamento de determinada variável no tempo ou no espaço (ASSIS et al., 1996) e tem sido considerada adequada ao estudo de séries temporais de elementos meteorológicos (SILVA\& FERNANDES, 1991; MEZZOMO et al., 2007; JOÃO, 2009). Os fundamentos teóricos e o método de utilização da análise harmônica para o estudo de séries temporais foram apresentados por AMARAL (1968).

No Estado do Rio Grande do Sul, a análise harmônica tem sido utilizada em séries temporais de temperaturas mínimas pentadais e mínimas absolutas pentadais de Pelotas do período de 1900 a 1987 (SILVA \& FERNANDES, 1991), de temperaturas mínimas médias trimestrais de 40 estações meteorológicas do período de 1913 a 2002 (MEZZOMO et al., 2007) e de temperaturas máxima e mínima médias mensais de 10 estações meteorológicas do período de 1918 e 2007 (JO ̃̃O, 2009) e, de maneira geral, os modelos revelaramse adequados. Nesses estudos, parte do período foi utilizada para a estimação dos coeficientes do modelo harmônico e parte para a validação do modelo. Embora os locais e o período desse estudo tenham sido avaliados em MEZZOMO et al. (2007), quanto à temperatura mínima média trimestral, estudos de séries temporais de temperatura do ar média mensal do Estado do Rio Grande do Sul, por meio de análise harmônica, não foram encontrados na literatura. Assim, o estudo da temperatura média, em uma escala mais detalhada (mensal), pode trazer contribuições importantes. Assim, o objetivo deste trabalho foi ajustar o modelo harmônico para a estimativa da temperatura do ar média mensal em 37 locais do Estado do Rio Grande do Sul, sendo utilizados os dados de 1931 a 1990 para estimação dos coeficientes do modelo harmônico e os de 1991 a 2000 para a validação do modelo.

\section{MATERIAL E MÉTODOS}

Foram utilizados os dados de temperatura do ar de 37 estações agrometeorológicas, localizadas em 37 locais do Estado do Rio Grande do Sul (RS) (Tabela 1). Os dados foram obtidos do Banco de Dados do Centro de Meteorologia Aplicada, da Fundação Estadual de Pesquisa Agropecuária - FEPAGRO/SCTRS e do Instituto Nacional de Meteorologia - INMET Porto Alegre-RS. Foram coletados no período de 1931 a 2000, totalizando 70 anos de observações. Em cada local e ano, a partir dos dados diários de temperatura mínima do ar (Tmin) e de temperatura máxima do ar (Tmax), calculou-se a temperatura média do ar por meio da expressão (Tmin+Tmax)/2. Em seguida, com os valores diários de temperatura média do ar, calculou-se a média mensal de temperatura, em ${ }^{\circ} \mathrm{C}$, de cada um dos 12 meses do ano, (janeiro, fevereiro, ..., dezembro), formando 444 séries temporais (37 locais x 12 meses) de temperatura do ar média mensal. Os dados de 1931 a 1990 (60 anos) foram utilizados para estimar os coeficientes do modelo harmônico, e os de 1991 a 2000 (10 anos), para a validação do modelo.

Em cada uma das 444 séries temporais de temperatura do ar média mensal do período de 1931 a 1990 (60 anos), foi verificada a normalidade dos dados, por meio do teste de Kolmogorov-Smirnov (SIEGEL \& CASTELLAN JÚNIOR, 2006). Em seguida, aplicou-se o teste de Bartlett (STEEL et al., 1997) aos dados temperatura do ar média mensal, para verificar a homogeneidade de variâncias entre os meses do ano (12 variâncias - variabilidade temporal), em cada local (37 testes).

A seguir, em cada local, os dados foram dispostos em ordem cronológica, sendo obtidas 37 séries temporais (37 locais), com 720 observações (60 anos x 12 meses). O método das duplas massas foi aplicado nessas 37 séries temporais e revelou homogeneidade dos dados nos 37 locais, o que confere credibilidade desses dados para análises de séries temporais. Em seguida, em cada uma dessas séries, verificou-se a tendência da temperatura do ar média mensal, ao longo do período de 1931 a 1990 (720 observações), por meio do ajuste de uma regressão linear simples dada por $\mathrm{Y}_{\mathrm{i}}=\mathrm{a}+\mathrm{bX} \mathrm{X}_{\mathrm{i}}$ em que $\mathrm{Y}_{\mathrm{i}}$ é a temperatura do ar média mensal (observada), a é o coeficiente linear, b é o coeficiente angular e $\mathrm{X}_{\mathrm{i}}$ é tempo, disposto em ordem cronológica, em que $\mathrm{i}=1$ (janeiro de 1931), 2 (fevereiro de 1931), ..., 720 (dezembro de 1990). 
Tabela 1 - P-valor do teste de normalidade (Kolmogorov-Smirnov) em relação a 444 séries temporais de temperatura do ar média mensal, em ${ }^{\circ} \mathrm{C}$, do período de 1931 a 1990, em locais do Estado do Rio Grande do Sul.

\begin{tabular}{|c|c|c|c|c|c|c|c|c|c|c|c|c|}
\hline \multirow{2}{*}{ Local } & \multirow[b]{2}{*}{ Jan } & \multirow[b]{2}{*}{$\mathrm{Fev}$} & \multirow[b]{2}{*}{ Mar } & \multirow[b]{2}{*}{ Abr } & \multirow[b]{2}{*}{ Mai } & \multirow[b]{2}{*}{ Jun } & \multirow[b]{2}{*}{ Jul } & \multirow[b]{2}{*}{ Ago } & \multirow[b]{2}{*}{ Set } & \multirow[b]{2}{*}{ Out } & \multirow[b]{2}{*}{ Nov } & \multirow[b]{2}{*}{ Dez } \\
\hline & & & & & & & & & & & & \\
\hline Alegrete & 0,996 & 0,811 & 0,736 & 0,963 & 0,627 & 0,762 & 1,000 & 0,782 & 0,382 & 0,697 & 0,718 & 0,521 \\
\hline Bagé & 0,786 & 0,788 & 0,804 & 0,682 & 0,769 & 0,798 & 0,979 & 0,871 & 0,452 & 0,736 & 0,951 & 0,732 \\
\hline Bento Gonçalves & 0,934 & 0,689 & 0,964 & 0,864 & 0,786 & 0,369 & 0,710 & 1,000 & 0,611 & 0,140 & 0,776 & 0,997 \\
\hline Bom Jesus & 0,088 & 0,900 & 0,907 & 0,971 & 0,907 & 0,600 & 0,745 & 0,804 & 0,951 & 0,544 & 0,939 & 0,526 \\
\hline Caçapava do Sul & 0,913 & 0,939 & 0,953 & 0,970 & 0,795 & 0,942 & 0,825 & 0,366 & 0,800 & 0,641 & 0,361 & 0,660 \\
\hline Cachoeira do Sul & 0,701 & 0,155 & 0,646 & 0,901 & 0,983 & 0,977 & 0,801 & 0,996 & 0,905 & 0,982 & 0,983 & 0,577 \\
\hline Caxias do Sul & 0,771 & 0,819 & 0,929 & 0,398 & 0,937 & 0,371 & 0,940 & 0,979 & 0,622 & 0,343 & 0,957 & 0,986 \\
\hline Cruz Alta & 0,973 & 0,584 & 0,961 & 0,891 & 0,699 & 0,600 & 0,892 & 0,974 & 0,887 & 0,726 & 0,792 & 0,832 \\
\hline Encruzilhada do Sul & 0,844 & 0,776 & 0,823 & 0,897 & 0,748 & 0,560 & 0,976 & 0,826 & 0,970 & 0,967 & 0,439 & 0,797 \\
\hline Farroupilha & 0,730 & 0,715 & 0,949 & 0,416 & 0,706 & 0,479 & 0,690 & 0,676 & 0,562 & 0,454 & 0,774 & 0,462 \\
\hline Guaporé & 0,680 & 0,548 & 0,849 & 0,788 & 0,788 & 0,628 & 0,760 & 0,600 & 0,767 & 0,247 & 0,931 & 0,952 \\
\hline Ijuí & 0,878 & 0,997 & 0,774 & 0,808 & 0,612 & 0,661 & 0,934 & 0,990 & 0,835 & 0,594 & 0,520 & 0,815 \\
\hline Iraí & 0,825 & 0,398 & 0,978 & 0,586 & 0,989 & 0,704 & 0,727 & 0,991 & 0,925 & 0,391 & 0,706 & 0,406 \\
\hline Júlio de Castilhos & 0,663 & 0,978 & 0,982 & 0,810 & 0,955 & 0,578 & 0,950 & 0,952 & 0,940 & 0,194 & 0,645 & 0,797 \\
\hline Lagoa Vermelha & 0,523 & 0,967 & 0,976 & 0,942 & 0,777 & 0,885 & 0,915 & 0,665 & 0,838 & 0,270 & 0,982 & 0,585 \\
\hline Osório & 0,909 & 0,891 & 0,699 & 0,719 & 0,982 & 0,653 & 0,846 & 0,534 & 0,725 & 0,351 & 0,998 & 0,536 \\
\hline Palmeira das Missões & 0,559 & 0,984 & 0,998 & 0,184 & 0,817 & 0,575 & 0,600 & 0,451 & 0,440 & 0,804 & 0,911 & 0,709 \\
\hline Passo Fundo & 0,493 & 0,746 & 0,731 & 0,412 & 0,697 & 0,451 & 0,885 & 0,839 & 0,889 & 0,453 & 0,136 & 0,750 \\
\hline Pelotas & 0,886 & 0,707 & 0,374 & 0,891 & 0,976 & 0,792 & 0,763 & 0,991 & 0,412 & 0,429 & 0,321 & 0,453 \\
\hline Porto Alegre & 0,832 & 0,946 & 0,338 & 0,996 & 0,846 & 0,448 & 0,966 & 0,988 & 0,977 & 0,117 & 0,646 & 0,758 \\
\hline Rio Grande & 0,981 & 0,896 & 0,916 & 0,999 & 0,894 & 0,556 & 0,978 & 0,973 & 0,993 & 0,687 & 0,755 & 0,823 \\
\hline Santa Maria & 0,975 & 0,742 & 0,662 & 0,893 & 0,635 & 0,864 & 0,840 & 0,875 & 0,500 & 0,999 & 0,353 & 0,710 \\
\hline Santana do Livramento & 0,939 & 0,933 & 0,744 & 0,762 & 0,667 & 0,658 & 0,994 & 0,965 & 0,655 & 0,538 & 0,956 & 0,796 \\
\hline Santa Rosa & 0,556 & 0,978 & 0,988 & 0,821 & 0,571 & 0,728 & 0,996 & 0,998 & 0,627 & 0,680 & 0,868 & 0,509 \\
\hline Santa Vitória do Palmar & 0,961 & 0,853 & 0,691 & 0,730 & 0,817 & 0,716 & 0,888 & 0,310 & 0,877 & 0,709 & 0,386 & 0,505 \\
\hline Santiago & 0,347 & 0,984 & 0,897 & 0,491 & 0,541 & 0,555 & 0,985 & 0,916 & 0,960 & 0,598 & 0,848 & 0,672 \\
\hline Santo Ângelo & 0,983 & 0,869 & 0,580 & 0,865 & 0,703 & 0,773 & 0,920 & 0,930 & 0,730 & 0,162 & 0,910 & 0,896 \\
\hline São Borja & 0,839 & 0,647 & 0,839 & 0,894 & 0,640 & 0,903 & 0,690 & 0,833 & 0,627 & 0,786 & 0,621 & 0,589 \\
\hline São Francisco de Paula & 0,930 & 0,933 & 0,776 & 0,958 & 0,558 & 0,684 & 0,905 & 0,877 & 0,709 & 0,953 & 0,961 & 0,330 \\
\hline São Gabriel & 0,798 & 0,617 & 0,810 & 0,907 & 0,615 & 0,857 & 0,804 & 0,581 & 0,787 & 0,596 & 0,399 & 0,863 \\
\hline São Luiz Gonzaga & 0,818 & 0,842 & 0,958 & 0,970 & 0,814 & 0,827 & 0,984 & 0,908 & 0,851 & 0,971 & 0,591 & 0,277 \\
\hline Tapes & 0,782 & 0,839 & 0,881 & 0,723 & 0,936 & 0,375 & 0,912 & 0,668 & 0,973 & 0,426 & 0,883 & 0,520 \\
\hline Taquari & 0,752 & 0,738 & 0,751 & 0,824 & 0,573 & 0,916 & 0,470 & 0,650 & 0,953 & 0,645 & 0,832 & 0,771 \\
\hline Torres & 0,906 & 0,860 & 0,999 & 0,716 & 0,825 & 0,684 & 0,652 & 0,884 & 0,807 & 0,537 & 0,978 & 0,893 \\
\hline Uruguaiana & 0,298 & 0,608 & 0,880 & 0,594 & 0,449 & 0,940 & 0,951 & 0,965 & 0,417 & 0,876 & 0,053 & 0,347 \\
\hline Vacaria & 0,745 & 0,985 & 0,925 & 0,967 & 0,535 & 0,671 & 0,689 & 0,804 & 0,231 & 0,475 & 0,977 & 0,486 \\
\hline Veranópolis & 0,964 & 0,971 & 0,965 & 0,459 & 0,945 & 0,438 & 0,809 & 0,998 & 0,878 & 0,336 & 0,984 & 0,614 \\
\hline
\end{tabular}

Nesse estudo, em particular, independentemente da significância do teste t de Student para testar as hipóteses $\mathrm{H}_{0}: \mathrm{b}=0$ versus $\mathrm{H}_{1}: \mathrm{b} \neq 0$, foi assumido que a estimativa do coeficiente angular (b) da regressão linear representa a tendência de acréscimo (se positivo) ou de decréscimo (se negativo) da temperatura do ar média mensal, em ${ }^{\circ} \mathrm{C}$, por mês. Depois disso, a fim de serem obtidas séries temporais adequadas para a análise harmônica, em cada uma das 37 séries temporais, foi calculada a temperatura do ar média mensal livre de tendência $\left(\mathrm{Y}_{\mathrm{ci}}\right)$, por meio da equação $\mathrm{Y}_{\mathrm{ci}}=\mathrm{Y}_{\mathrm{i}}-\mathrm{b} \mathrm{X}_{\mathrm{i}}$.
A seguir, para cada uma das 37 séries temporais de $\mathrm{Y}_{\mathrm{ci}}$, calculou-se a média de temperatura do ar média mensal de cada um dos 12 meses do ano, referente ao período de 1931 a 1990. Essas 12 médias, obtidas a partir de 60 anos de observações, foram utilizadas para análise harmônica conforme procedimentos descritos em AMARAL (1968) e ASSIS et al. (1996), considerando o mês como unidade de tempo. Assim, foi considerado o período igual a $12 \mathrm{e}$ foram computadas seis ondas harmônicas, com seis termos em co-senos e cinco em senos. Nos 37 locais, 
foi selecionado o modelo com base na primeira (onda anual) e na segunda harmônica (onda semestral) pelo fato de as duas ondas explicarem aproximadamente $100 \%\left(\mathrm{R}^{2} \geq 0,9867\right)$ da variação total da série de dados analisados.

Em cada um dos 37 locais, foram estimados (previstos) os valores de temperatura do ar média mensal $\left(\hat{\mathrm{Y}}_{\mathrm{i}}\right)$, para o período de 1991 a $2000(120$ observações), com base no seguinte modelo: $\hat{\mathrm{Y}}=\mathrm{P}_{0}+\mathrm{P}_{1}$ $\cos \left(30^{\circ} \mathrm{t}\right)+\mathrm{Q}_{1} \operatorname{sen}\left(30^{\circ} \mathrm{t}\right)+\mathrm{P}_{2} \cos \left(60^{\circ} \mathrm{t}\right)+\mathrm{Q}_{2} \operatorname{sen}\left(60^{\circ} \mathrm{t}\right)+\mathrm{b}$ (720+i), em que $\mathrm{P}_{0}$ é a média dos 12 valores da série (12 meses), $\mathrm{P}_{1}$ e $\mathrm{Q}_{1}$ são os coeficientes da primeira harmônica, $\mathrm{P}_{2}$ e $\mathrm{Q}_{2}$ são os coeficientes da segunda harmônica, $\mathrm{b}$ é a estimativa da tendência, $\mathrm{t}=0$ (janeiro), 1 (fevereiro), ..., 11(dezembro), 720 é o número de observações (60 anos x 12 meses) que foram utilizadas para a estimativa de $\mathrm{b}$ (tendência) e o índice i é o número de meses à frente do modelo, ou seja, $\mathrm{i}=1$ (janeiro de 1991), 2 (fevereiro de 1991), ..., 120 (dezembro de 2000).

A validação do modelo de estimativa de temperatura do ar média mensal, em cada um dos 37 locais, foi realizada com base nos 120 valores estimados pelo modelo ( $\hat{\mathrm{Y}}_{\mathrm{i}}$ ) e os 120 valores observados $\left(\mathrm{Y}_{\mathrm{i}}\right)$. Inicialmente, foi ajustada uma regressão linear simples $\left(\hat{\mathrm{Y}}_{\mathrm{i}}=\mathrm{a}+\mathrm{b} \mathrm{Y}_{\mathrm{i}}\right)$ e, por meio do teste t de Student, a 5\% de probabilidade de erro, foram testadas as hipóteses $\mathrm{H}_{0}: \mathrm{a}=0$ versus $\mathrm{H}_{1}: \mathrm{a} \neq 0$ e $\mathrm{H}_{0}: \mathrm{b}=1$ versus $\mathrm{H}_{1} \quad \mathrm{~b} \neq 1$. Foram calculados os coeficientes de correlação linear de Pearson ( $r)$ e de determinação $\left(R^{2}\right)$ entre $\hat{\mathrm{Y}}_{\mathrm{i}}$ e $\mathrm{Y}_{\mathrm{i}}$. A raiz do quadrado médio do erro (RQME) e o índice d de Willmott (WILLMOTT, $1981)$ foram calculados, respectivamente, por

$R Q M E=\sqrt{\frac{\sum_{i=1}^{n}\left(\hat{Y}_{i}-Y_{i}\right)^{2}}{n}} \quad$ e $\quad d=1-\left[\frac{\sum_{i=1}^{n}\left(\hat{Y}_{i}-Y_{i}\right)^{2}}{\sum_{i=1}^{n}\left(\left|\hat{Y}_{i}-\bar{Y}\right|+\left|Y_{i}-\bar{Y}\right|\right)^{2}}\right]$, em que $\hat{Y}_{i}$ são os valores estimados, Yi são os valores observados, $\bar{Y}$ é a média dos valores observados e n é o número de observações $(n=120)$. Em seguida, foi calculado o índice CS de CAMARGO \& SENTELHAS (1997) pelo produto entre r e d (CS=r*d).

\section{RESULTADOS E DISCUSSÃO}

Nas 444 séries temporais (37 locais x 12 meses) de temperatura do ar média mensal, o p-valor do teste Kolmogorov-Smirnov oscilou entre 0,053 (mês de novembro em Uruguaiana) e 1,000 (mês de julho em Alegrete e mês de agosto em Bento Gonçalves), e a média dos 444 p-valores foi 0,748 (Tabela 1). Esses resultados evidenciam boa aderência dos dados de temperatura do ar média mensal à distribuição normal, pois quanto mais próximo de um for o p-valor, maior é a evidência de que a série seja normal. Assim, em cada mês e local, pode-se inferir que, em $50 \%$ dos anos, os valores são inferiores à média e em $50 \%$ dos anos são superiores. Portanto, a média é uma medida de tendência central adequada para representar a temperatura do ar média mensal. Esse fato caracteriza esse conjunto de dados como adequado para geração de modelos por meio da análise harmônica. Estudo prévio constatou boa aderência dos dados de temperatura máxima do ar média decendial de 23 municípios do Rio Grande do Sul à distribuição normal (CARGNELUTTI FILHO et al., 2008a).

O teste de Bartlett (STEEL et al., 1997) aplicado entre as variâncias dos 12 meses, em cada local, constatou que estas foram heterogêneas $(\mathrm{P} \leq 0,05)$ em todos os locais (Tabela 2). De maneira geral, nos 37 locais, menores variâncias de temperatura do ar média mensal ocorreram nos meses inicial (janeiro) e final (dezembro), com aumento gradativo em direção aos meses centrais do ano (maio, junho e julho). Esses resultados sugerem que os valores estimados por meio dos modelos harmônicos sejam mais precisos nos meses com menor variância (meses iniciais e finais do ano) e menos precisos nos meses com maior variância (meses centrais do ano). Modelos empíricos para a estimativa de temperaturas do ar mínima decendial (CARGNELUTTI FILHO et al., 2006) e média decendial (CARGNELUTTI FILHO et al., 2008b), no Estado do Rio Grande do Sul, em função de coordenadas geográficas, também, apresentaram os maiores valores de $\mathrm{R}^{2}$ (melhores ajustes do modelo) nos primeiros e nos últimos meses do ano, com diminuição gradativa em direção aos meses centrais do ano. Portanto, a presença de heterogeneidade de variâncias sugere maior precisão nas estimativas, nos meses iniciais e finais do ano, com diminuição gradativa para os meses centrais. Transformações de dados podem ser utilizadas a fim de homogeneizar as variâncias. No entanto, nesse estudo em particular, a boa aderência dos dados à distribuição normal oferece credibilidade aos modelos gerados a partir da análise harmônica.

O valor do coeficiente angular da regressão linear das 37 séries de temperatura do ar média mensal oscilou entre -0,00103415, em Santo Ângelo, e 0,00187262, em São Francisco de Paula, e a média das 37 estimativas foi de 0,00031137 (Tabela 3 ). Isso significa decréscimo de $0,00103415^{\circ} \mathrm{C}$ por mês, em Santo Ângelo, e acréscimo de $0,00187262^{\circ} \mathrm{C}$ por mês, em São Francisco de Paula. Houve tendência decrescente em 12 locais $(32,43 \%)$ e crescente em 25 locais $(67,57 \%)$. É importante estudar mais detalhadamente a tendência, pois, neste estudo, em particular, o foco foi apenas estimar o b (tendência) para incorporá-lo no modelo harmônico, a fim de serem obtidas melhores estimativas. 
Tabela 2 - Estimativa da variância da temperatura do ar média mensal com os dados do período de 1931 a 1990 e valor calculado do teste de Bartlett das variâncias entre os meses em cada local ( $\left.\chi_{\text {calc }}^{2}\right)$ do Estado do Rio Grande do Sul.

\begin{tabular}{|c|c|c|c|c|c|c|c|c|c|c|c|c|c|}
\hline \multirow{2}{*}{ Local } & & \multirow[b]{2}{*}{ Abr } & \multirow[b]{2}{*}{ Mai } & \multirow[b]{2}{*}{ Jun } & \multirow[b]{2}{*}{ Jul } & \multirow[b]{2}{*}{ Ago } & \multirow[b]{2}{*}{ Set } & \multirow[b]{2}{*}{ Out } & \multirow[b]{2}{*}{ Nov } & \multirow[b]{2}{*}{ Dez } & \multirow[t]{2}{*}{$\chi_{\text {calc }}^{2}$} \\
\hline & Jan & Fev & Mar & & & & & & & & & & \\
\hline Alegrete & 0,692 & 1,167 & 1,224 & 1,780 & 3,316 & 2,661 & 3,051 & 2,117 & 1,627 & 1,892 & 1,721 & 1,276 & $60,83^{*}$ \\
\hline Bagé & 0,773 & 1,300 & 1,116 & 1,586 & 2,923 & 2,327 & 2,880 & 2,059 & 1,567 & 1,765 & 1,462 & 1,176 & $49,58 *$ \\
\hline Bento Gonçalves & 0,462 & 0,974 & 0,995 & 1,913 & 2,952 & 2,108 & 2,969 & 2,116 & 1,884 & 1,830 & 1,013 & 0,959 & $88,81^{*}$ \\
\hline Bom Jesus & 1,013 & 0,931 & 0,935 & 1,762 & 2,853 & 2,163 & 2,843 & 2,002 & 1,573 & 1,824 & 1,176 & 0,719 & $65,68 *$ \\
\hline Caçapava do Sul & 0,708 & 1,102 & 1,202 & 1,876 & 2,623 & 1,914 & 2,873 & 2,042 & 1,492 & 2,173 & 1,446 & 1,522 & $46,16^{*}$ \\
\hline Cachoeira do Sul & 0,744 & 1,170 & 1,024 & 2,202 & 3,718 & 2,649 & 2,876 & 1,899 & 2,007 & 1,958 & 1,421 & 0,978 & $74,08^{*}$ \\
\hline Caxias do Sul & 0,615 & 1,066 & 1,141 & 1,783 & 2,617 & 2,022 & 2,958 & 2,360 & 1,666 & 1,853 & 1,127 & 0,776 & $72,11^{*}$ \\
\hline Cruz Alta & 0,681 & 0,895 & 1,176 & 1,599 & 2,742 & 2,296 & 3,094 & 2,237 & 1,867 & 1,695 & 1,140 & 1,054 & $68,38 *$ \\
\hline Encruzilhada do Sul & 0,632 & 1,175 & 1,369 & 1,568 & 2,571 & 1,957 & 2,458 & 1,874 & 1,682 & 1,731 & 1,173 & 1,106 & $44,64^{*}$ \\
\hline Farroupilha & 0,551 & 1,243 & 1,128 & 1,887 & 2,788 & 2,023 & 2,635 & 2,073 & 1,613 & 1,720 & 0,948 & 0,732 & $74,36^{*}$ \\
\hline Guaporé & 0,521 & 0,735 & 1,007 & 1,912 & 2,553 & 2,388 & 3,083 & 2,154 & 1,549 & 1,643 & 0,962 & 0,949 & $91,44^{*}$ \\
\hline Ijuí & 0,801 & 0,868 & 1,276 & 1,724 & 2,897 & 2,628 & 3,536 & 2,120 & 1,823 & 1,712 & 1,039 & 1,090 & $74,72^{*}$ \\
\hline Iraí & 0,837 & 1,005 & 0,945 & 2,160 & 2,629 & 2,057 & 3,372 & 2,243 & 1,623 & 2,032 & 1,477 & 1,031 & $63,47 *$ \\
\hline Júlio de Castilhos & 0,789 & 0,794 & 1,103 & 1,766 & 2,357 & 2,058 & 2,811 & 2,278 & 1,542 & 1,736 & 1,127 & 1,276 & $54,37^{*}$ \\
\hline Lagoa Vermelha & 0,572 & 0,756 & 0,975 & 1,771 & 2,458 & 1,928 & 2,832 & 2,300 & 1,593 & 1,326 & 0,952 & 0,921 & $80,04^{*}$ \\
\hline Osório & 0,829 & 1,175 & 1,030 & 1,658 & 2,652 & 2,303 & 2,849 & 2,068 & 1,671 & 1,796 & 0,877 & 0,921 & $61,51^{*}$ \\
\hline Palmeira das Missões & 0,769 & 0,812 & 1,337 & 1,603 & 2,480 & 2,023 & 2,579 & 1,781 & 1,258 & 1,705 & 1,015 & 1,147 & $50,22^{*}$ \\
\hline Passo Fundo & 0,606 & 0,782 & 0,983 & 1,810 & 2,557 & 2,002 & 2,648 & 2,240 & 1,663 & 1,589 & 0,835 & 0,811 & $81,34^{*}$ \\
\hline Pelotas & 0,844 & 0,864 & 1,278 & 1,511 & 2,843 & 2,105 & 1,997 & 1,451 & 1,298 & 1,666 & 1,050 & 0,924 & $47,82^{*}$ \\
\hline Porto Alegre & 0,597 & 0,872 & 1,075 & 1,458 & 2,688 & 2,156 & 2,534 & 1,510 & 1,407 & 1,390 & 0,804 & 0,856 & $72,13^{*}$ \\
\hline Rio Grande & 0,576 & 0,718 & 0,934 & 1,159 & 2,458 & 2,159 & 1,971 & 0,932 & 1,040 & 1,251 & 0,737 & 0,667 & $77,90^{*}$ \\
\hline Santa Maria & 0,694 & 0,924 & 1,099 & 1,649 & 3,381 & 3,090 & 3,177 & 2,151 & 1,742 & 0,912 & 1,269 & 0,893 & $99,59^{*}$ \\
\hline Santana do Livramento & 1,191 & 0,959 & 1,534 & 1,788 & 2,880 & 2,394 & 2,354 & 1,942 & 1,517 & 1,694 & 1,572 & 0,969 & $37,54^{*}$ \\
\hline Santa Rosa & 0,919 & 0,966 & 1,151 & 1,783 & 2,908 & 2,324 & 3,102 & 2,287 & 1,906 & 1,629 & 1,218 & 0,923 & $62,26^{*}$ \\
\hline Santa Vitória do Palmar & 0,712 & 1,153 & 1,014 & 1,142 & 2,320 & 1,750 & 1,859 & 0,981 & 1,043 & 1,202 & 0,945 & 0,969 & $38,72^{*}$ \\
\hline Santiago & 1,156 & 1,333 & 1,356 & 1,836 & 2,982 & 2,261 & 3,134 & 2,374 & 1,574 & 2,162 & 1,009 & 1,170 & $47,56^{*}$ \\
\hline Santo Ângelo & 0,633 & 0,967 & 1,418 & 1,891 & 2,825 & 2,729 & 3,390 & 2,887 & 2,182 & 1,612 & 1,341 & 1,211 & $75,68^{*}$ \\
\hline São Borja & 1,077 & 1,103 & 1,595 & 1,520 & 3,304 & 3,189 & 3,164 & 2,569 & 1,781 & 2,011 & 1,625 & 1,590 & $49,62 *$ \\
\hline São Francisco de Paula & 0,710 & 1,233 & 1,223 & 2,077 & 2,601 & 1,824 & 2,407 & 1,996 & 1,715 & 2,056 & 1,276 & 1,158 & $42,10^{*}$ \\
\hline São Gabriel & 0,723 & 1,196 & 1,607 & 1,754 & 3,225 & 2,780 & 2,726 & 1,977 & 1,479 & 1,732 & 1,382 & 0,999 & $59,24^{*}$ \\
\hline São Luiz Gonzaga & 0,823 & 0,926 & 1,454 & 1,716 & 2,648 & 2,402 & 2,898 & 2,319 & 1,560 & 1,891 & 1,423 & 1,237 & $49,21^{*}$ \\
\hline Tapes & 0,701 & 0,877 & 1,024 & 1,130 & 2,756 & 2,377 & 2,525 & 1,411 & 1,243 & 1,438 & 1,114 & 1,109 & $62,47^{*}$ \\
\hline Taquari & 0,648 & 0,950 & 0,938 & 1,553 & 2,997 & 2,402 & 2,652 & 1,863 & 1,470 & 1,586 & 0,970 & 0,842 & $77,76^{*}$ \\
\hline Torres & 0,600 & 0,715 & 0,709 & 1,000 & 1,807 & 1,799 & 1,855 & 1,279 & 1,150 & 0,925 & 0,652 & 0,758 & $57,38^{*}$ \\
\hline Uruguaiana & 0,941 & 1,248 & 1,200 & 1,280 & 2,857 & 2,858 & 3,243 & 1,920 & 1,380 & 1,640 & 1,275 & 1,240 & $56,79^{*}$ \\
\hline Vacaria & 0,659 & 0,934 & 1,226 & 1,712 & 2,594 & 2,113 & 2,951 & 2,102 & 1,572 & 1,602 & 0,863 & 1,001 & $68,07^{*}$ \\
\hline Veranópolis & 0,542 & 1,109 & 1,127 & 1,885 & 2,701 & 2,044 & 2,986 & 2,139 & 1,731 & 1,865 & 1,040 & 0,725 & $80,32^{*}$ \\
\hline
\end{tabular}

* Significativo a 5\% de probabilidade de erro pelo teste de Bartlett (variâncias heterogêneas).

O coeficiente de determinação $\left(\mathrm{R}^{2}\right)$ do modelo harmônico, com base na primeira (onda anual) e na segunda harmônica (onda semestral), foi elevado em todos os locais $\left(\mathrm{R}^{2}=0,9867\right)$, sugerindo confiabilidade nas previsões futuras (Tabela 3). No entanto, em 28 locais, o coeficiente linear (a) da regressão linear de $\hat{Y}_{i}$ em função de $Y_{i}$ diferiu de zero $(\mathrm{P} \leq 0,05)$ e em 29 locais o coeficiente angular (b) diferiu de um $(\mathrm{P} \leq 0,05)$ (Tabela 4), podendo isso, provavelmente, ser atribuído à maior variabilidade dos dados de temperatura do ar média mensal, nos meses centrais do ano (Tabela 2). Assim, quando os valores observados são baixos, os modelos estimam valores um pouco maiores e vice-versa. Esse comportamento foi evidenciado em praticamente todos esses locais e é 
Tabela 3 - Estimativa dos coeficientes $\mathrm{P}_{0}, \mathrm{P}_{1}, \mathrm{Q}_{1}, \mathrm{P}_{2}$ e $\mathrm{Q}_{2}$ obtidos a partir da análise harmônica da temperatura do ar média mensal, com os dados do período de 1931 a 1990 . Estimativa da tendência linear (b) e do coeficiente de determinação $\left(\mathrm{R}^{2}\right)$ do modelo ${ }^{(1)}$ envolvendo duas harmônicas (anual e semestral), em locais do Estado do Rio Grande do Sul.

\begin{tabular}{|c|c|c|c|c|c|c|c|}
\hline Local & $\mathrm{P}_{0}$ & $\mathrm{P}_{1}$ & $\mathrm{Q}_{1}$ & $\mathrm{P}_{2}$ & $\mathrm{Q}_{2}$ & $\mathrm{~b}$ & $\mathrm{R}^{2}$ \\
\hline Alegrete & 19,135828 & 5,606824 & 0,285129 & 0,150536 & 0,522108 & 0,00086263 & 0,9983 \\
\hline Bagé & 18,408003 & 5,629698 & 0,432119 & 0,167033 & 0,488014 & 0,00043569 & 0,9985 \\
\hline Bento Gonçalves & 17,448298 & 4,663921 & 0,232079 & 0,104410 & 0,610008 & 0,00059782 & 0,9981 \\
\hline Bom Jesus & 15,952353 & 4,326262 & 0,350543 & 0,101316 & 0,561564 & $-0,00067699$ & 0,9990 \\
\hline Caçapava do Sul & 17,314090 & 5,297740 & 0,506865 & 0,047996 & 0,535461 & 0,00114844 & 0,9957 \\
\hline Cachoeira do Sul & 19,695684 & 5,582269 & 0,133312 & 0,190099 & 0,407997 & $-0,00093604$ & 0,9988 \\
\hline Caxias do Sul & 16,752470 & 4,460306 & 0,340953 & 0,169975 & 0,515555 & 0,00094730 & 0,9983 \\
\hline Cruz Alta & 18,950001 & 4,905885 & 0,174467 & 0,092045 & 0,426956 & 0,00015615 & 0,9979 \\
\hline Encruzilhada do Sul & 17,114790 & 5,081794 & 0,587990 & 0,070371 & 0,481023 & 0,00130204 & 0,9977 \\
\hline Farroupilha & 16,667272 & 4,649794 & 0,140081 & 0,112866 & 0,498454 & 0,00035227 & 0,9979 \\
\hline Guaporé & 18,271820 & 5,026737 & 0,100332 & 0,104921 & 0,498766 & 0,00026136 & 0,9996 \\
\hline Ijuí & 19,923024 & 5,107903 & 0,031894 & 0,145718 & 0,403127 & 0,00028041 & 0,9981 \\
\hline Iraí & 19,699777 & 5,283411 & $-0,007988$ & $-0,005456$ & 0,639564 & 0,00175940 & 0,9978 \\
\hline Júlio de Castilhos & 17,847402 & 5,054403 & 0,304376 & 0,070897 & 0,461245 & 0,00102909 & 0,9984 \\
\hline Lagoa Vermelha & 17,548174 & 4,691778 & 0,142013 & $-0,007081$ & 0,546273 & $-0,00019938$ & 0,9981 \\
\hline Osório & 19,046083 & 4,240583 & 0,575387 & $-0,019768$ & 0,542678 & 0,00055118 & 0,9984 \\
\hline Palmeira das Missões & 18,931941 & 4,830634 & 0,054728 & 0,012889 & 0,484012 & 0,00004882 & 0,9967 \\
\hline Passo Fundo & 18,180198 & 4,717410 & 0,038584 & 0,053681 & 0,456397 & 0,00035486 & 0,9978 \\
\hline Pelotas & 17,738696 & 5,256055 & 0,799985 & 0,078882 & 0,557420 & 0,00072235 & 0,9990 \\
\hline Porto Alegre & 20,067135 & 5,215925 & 0,551960 & 0,008486 & 0,554328 & 0,00016666 & 0,9991 \\
\hline Rio Grande & 18,524568 & 5,220091 & 0,988395 & $-0,096177$ & 0,485293 & 0,00015650 & 0,9992 \\
\hline Santa Maria & 19,710516 & 5,231628 & 0,339663 & 0,134196 & 0,518223 & $-0,00017865$ & 0,9981 \\
\hline Santana do Livramento & 18,361588 & 5,712143 & 0,300550 & 0,206594 & 0,467744 & $-0,00039229$ & 0,9991 \\
\hline Santa Rosa & 20,326132 & 5,307978 & $-0,124824$ & 0,095260 & 0,554008 & $-0,00019880$ & 0,9976 \\
\hline Santa Vitória do Palmar & 17,370874 & 5,368330 & 0,984156 & 0,094423 & 0,483855 & $-0,00012561$ & 0,9990 \\
\hline Santiago & 18,574923 & 5,306724 & 0,247346 & 0,167058 & 0,547910 & 0,00091960 & 0,9983 \\
\hline Santo Ângelo & 20,454840 & 5,238382 & $-0,021074$ & 0,070730 & 0,533653 & $-0,00103415$ & 0,9985 \\
\hline São Borja & 20,699289 & 5,479690 & 0,231733 & 0,146388 & 0,515163 & $-0,00027211$ & 0,9985 \\
\hline São Francisco de Paula & 14,880880 & 4,266514 & 0,397027 & 0,130609 & 0,512563 & 0,00187262 & 0,9984 \\
\hline São Gabriel & 19,135379 & 5,804268 & 0,309308 & 0,121869 & 0,547990 & 0,00021574 & 0,9988 \\
\hline São Luiz Gonzaga & 20,299088 & 5,285652 & 0,062468 & 0,111217 & 0,486120 & 0,00069620 & 0,9973 \\
\hline Tapes & 19,027489 & 5,312349 & 0,819001 & 0,001268 & 0,567085 & 0,00023986 & 0,9991 \\
\hline Taquari & 20,166268 & 5,337757 & 0,275499 & 0,129188 & 0,419217 & $-0,00084001$ & 0,9991 \\
\hline Torres & 18,128064 & 4,072320 & 1,101903 & $-0,084880$ & 0,418739 & 0,00144671 & 0,9990 \\
\hline Uruguaiana & 20,232317 & 5,948922 & 0,270392 & 0,160016 & 0,459863 & $-0,00014383$ & 0,9984 \\
\hline Vacaria & 16,496622 & 4,693199 & 0,116398 & 0,064170 & 0,584212 & $-0,00102465$ & 0,9978 \\
\hline Veranópolis & 16,818736 & 4,554380 & 0,191810 & 0,125277 & 0,505588 & 0,00101957 & 0,9983 \\
\hline
\end{tabular}

${ }^{(1)} \mathrm{Y}_{\mathrm{i}}=\mathrm{P}_{0}+\mathrm{P}_{1} \cos \left(30^{\circ} \mathrm{t}\right)+\mathrm{Q}_{1} \operatorname{sen}\left(30^{\circ} \mathrm{t}\right)+\mathrm{P}_{2} \cos \left(60^{\circ} \mathrm{t}\right)+\mathrm{Q}_{2} \operatorname{sen}\left(60^{\circ} \mathrm{t}\right)+\mathrm{b}(720+\mathrm{i})$, em que $\mathrm{Y}_{\mathrm{i}}$ é a estimativa da temperatura do ar média mensal, $\mathrm{P}_{0}$ é a média dos 12 valores da série (12 meses), $P_{1}$ e $Q_{1}$ são os coeficientes da primeira harmônica, $P_{2}$ e $Q_{2}$ são os coeficientes da segunda harmônica, $\mathrm{b}$ é a estimativa da tendência, $\mathrm{t}=0$ (janeiro), 1 (fevereiro), ..., 11(dezembro), 720 é o número de observações (60 anos x 12 meses) que foram utilizadas para a estimativa de b (tendência) e o índice i é o número de meses a frente do modelo, ou seja, i=1 (janeiro de 1991), 2 (fevereiro de 1991), ..., 120 (dezembro de 2000).

exemplificado com os dados de Alegrete (Figura 1). Então, num primeiro momento, para esses locais, poderse-ia, com base somente nesses coeficientes, desqualificar esses modelos. No entanto, isso deve ser visto com cautela, pois o modelo é válido dentro do intervalo de valores utilizados para a sua construção, e ainda extrapolações para temperaturas zero, que do ponto de vista prático dificilmente ocorrerão, não são adequadas. Além disso, os demais indicadores (Tabela 4) confirmam a adequabilidade dos modelos nesses locais. Nos demais locais, todos os indicadores foram favoráveis, ou seja, coeficiente linear (a) não diferente de zero, coeficiente angular (b) não diferente de um, coeficientes de correlação linear de Pearson (r) e de 
Tabela 4 - Coeficientes linear (a), angular (b), de correlação linear de Pearson (r) e de determinação ( $\mathrm{R}^{2}$ ) obtidos na regressão linear ajustada entre a temperatura do ar média mensal estimada (variável dependente) e a observada (variável independente). Raiz do quadrado médio do erro (RQME), índice d de Willmott (WILLMOTT, 1981) e índice CS de CAMARGO \& SENTELHAS (1997) calculados com base na temperatura do ar média mensal estimada e observada no período de janeiro de 1991 a dezembro de 2000, em locais do Estado do Rio Grande do Sul.

\begin{tabular}{|c|c|c|c|c|c|c|c|}
\hline Local & $a^{(1)}$ & $b^{(2)}$ & $r^{(3)}$ & $\mathrm{R}^{2}$ & RQME & d & $\mathrm{CS}$ \\
\hline Alegrete & $2,460 *$ & $0,884 *$ & $0,954 *$ & 0,911 & 1,302 & 0,974 & 0,930 \\
\hline Bagé & $1,053 *$ & $0,960 \mathrm{~ns}$ & $0,960 *$ & 0,923 & 1,167 & 0,978 & 0,940 \\
\hline Bento Gonçalves & $1,749 *$ & $0,898 *$ & $0,949 *$ & 0,902 & 1,102 & 0,973 & 0,924 \\
\hline Bom Jesus & $1,429 *$ & $0,894 *$ & $0,946 *$ & 0,896 & 1,080 & 0,970 & 0,918 \\
\hline Caçapava do Sul & $0,879 \mathrm{~ns}$ & $0,944 \mathrm{~ns}$ & $0,957 *$ & 0,917 & 1,114 & 0,978 & 0,936 \\
\hline Cachoeira do Sul & $-0,132 \mathrm{~ns}$ & $0,977 \mathrm{~ns}$ & $0,961 *$ & 0,924 & 1,233 & 0,975 & 0,937 \\
\hline Caxias do Sul & $2,013 *$ & $0,884 *$ & $0,941 *$ & 0,885 & 1,144 & 0,968 & 0,911 \\
\hline Cruz Alta & $1,963 *$ & $0,886 *$ & $0,952 *$ & 0,906 & 1,167 & 0,973 & 0,926 \\
\hline Encruzilhada do Sul & $2,050 *$ & $0,896 *$ & $0,955 *$ & 0,912 & 1,162 & 0,975 & 0,931 \\
\hline Farroupilha & $1,575 *$ & $0,893 *$ & $0,955 *$ & 0,912 & 1,077 & 0,974 & 0,931 \\
\hline Guaporé & $0,876 \mathrm{~ns}$ & $0,948 \mathrm{~ns}$ & $0,957 *$ & 0,916 & 1,052 & 0,978 & 0,936 \\
\hline Ijuí & $2,440 *$ & $0,882 *$ & $0,935 *$ & 0,874 & 1,362 & 0,965 & 0,903 \\
\hline Iraí & $1,419 *$ & $0,936 *$ & $0,956 *$ & 0,915 & 1,125 & 0,977 & 0,935 \\
\hline Júlio de Castilhos & $2,116 *$ & $0,887 *$ & $0,951 *$ & 0,905 & 1,188 & 0,973 & 0,926 \\
\hline Lagoa Vermelha & $0,934 \mathrm{~ns}$ & $0,946 \mathrm{~ns}$ & $0,940 *$ & 0,885 & 1,146 & 0,969 & 0,912 \\
\hline Osório & $3,217 *$ & $0,859 *$ & $0,951 *$ & 0,905 & 1,185 & 0,965 & 0,918 \\
\hline Palmeira das Missões & $1,888 *$ & $0,877 *$ & $0,948 *$ & 0,899 & 1,273 & 0,967 & 0,917 \\
\hline Passo Fundo & $1,702 *$ & $0,905 *$ & $0,951 *$ & 0,905 & 1,083 & 0,974 & 0,927 \\
\hline Pelotas & $1,845 *$ & $0,897 *$ & $0,942 *$ & 0,888 & 1,329 & 0,969 & 0,913 \\
\hline Porto Alegre & $1,451 *$ & $0,920 *$ & $0,962 *$ & 0,927 & 1,065 & 0,980 & 0,943 \\
\hline Rio Grande & $1,614 *$ & $0,919 *$ & $0,945 *$ & 0,894 & 1,268 & 0,971 & 0,919 \\
\hline Santa Maria & $2,013 *$ & $0,884 *$ & $0,954 *$ & 0,911 & 1,230 & 0,974 & 0,930 \\
\hline Santana do Livramento & $-0,146 \mathrm{~ns}$ & $0,970 \mathrm{~ns}$ & $0,959 *$ & 0,920 & 1,341 & 0,972 & 0,932 \\
\hline Santa Rosa & $0,937 \mathrm{~ns}$ & $0,932 *$ & $0,947 *$ & 0,897 & 1,323 & 0,969 & 0,917 \\
\hline Santa Vitória do Palmar & $1,310 *$ & $0,920 *$ & $0,971 *$ & 0,943 & 0,975 & 0,984 & 0,956 \\
\hline Santiago & $1,544 *$ & $0,920 *$ & $0,960 *$ & 0,922 & 1,099 & 0,979 & 0,940 \\
\hline Santo Ângelo & $1,221 \mathrm{~ns}$ & $0,932 \mathrm{~ns}$ & $0,938 *$ & 0,881 & 1,312 & 0,968 & 0,909 \\
\hline São Borja & $0,049 \mathrm{~ns}$ & $0,971 \mathrm{~ns}$ & $0,952 *$ & 0,908 & 1,310 & 0,970 & 0,925 \\
\hline São Francisco de Paula & $1,944 *$ & $0,885 *$ & $0,948 *$ & 0,899 & 1,041 & 0,972 & 0,921 \\
\hline São Gabriel & $1,514 *$ & $0,908 *$ & $0,954 *$ & 0,911 & 1,321 & 0,975 & 0,930 \\
\hline São Luiz Gonzaga & $0,685 \mathrm{~ns}$ & $0,938 *$ & $0,953 *$ & 0,908 & 1,319 & 0,969 & 0,923 \\
\hline Tapes & $1,186 *$ & $0,949 *$ & $0,969 *$ & 0,939 & 0,985 & 0,983 & 0,953 \\
\hline Taquari & $2,061 *$ & $0,906 *$ & $0,952 *$ & 0,907 & 1,239 & 0,974 & 0,928 \\
\hline Torres & $2,266 *$ & $0,865 *$ & $0,961 *$ & 0,923 & 1,016 & 0,973 & 0,935 \\
\hline Uruguaiana & $1,945 *$ & $0,929 *$ & $0,953 *$ & 0,910 & 1,424 & 0,971 & 0,927 \\
\hline Vacaria & $1,034 *$ & $0,891 *$ & $0,947 *$ & 0,897 & 1,364 & 0,960 & 0,909 \\
\hline Veranópolis & $2,269 *$ & $0,884 *$ & $0,945 *$ & 0,893 & 1,163 & 0,969 & 0,916 \\
\hline
\end{tabular}

(1) * Coeficiente linear difere de zero pelo teste t, com nível de 5\% de probabilidade. ns = Não significativo.

(2) * Coeficiente angular difere de um pelo teste t, com nível de $5 \%$ de probabilidade. ns = Não significativo.

(3) * Coeficiente de correlação difere de zero pelo teste t, com nível de $5 \%$ de probabilidade. ns = Não significativo.

determinação $\left(\mathrm{R}^{2}\right)$ próximos de um, raiz do quadrado médio do erro (RQME) próxima de zero e índices d de Willmott (WILLMOTT, 1981) e CS de CAMARGO \& SENTELHAS (1997) próximos de um. Portanto, de maneira geral, pode-se inferir que esses modelos podem ser utilizados para estimar a temperatura do ar média mensal nesses locais do Estado do Rio Grande do Sul.
Assim, em Alegrete, por exemplo, a estimativa da temperatura do ar média mensal ( $\hat{\mathrm{Y}}_{i}$ ) para o mês de janeiro de 1991 é $25,5151^{\circ} \mathrm{C}$ e é obtida por meio da equação $\hat{\mathrm{Y}}_{\mathrm{i}}=\mathrm{P}_{0}+\mathrm{P}_{1} \cos \left(30^{\circ} \mathrm{t}\right)+\mathrm{Q}_{1} \operatorname{sen}\left(30^{\circ} \mathrm{t}\right)+\mathrm{P}_{2}$ $\cos \left(60^{\circ} \mathrm{t}\right)+\mathrm{Q}_{2} \operatorname{sen}\left(60^{\circ} \mathrm{t}\right)+\mathrm{b}(720+\mathrm{i})$ (Tabela 3$)$, ou seja, $\hat{\mathrm{Y}}_{1}=19,135828+5,606824 \cos \left(30^{\circ} * 0\right)+0,285129 \operatorname{sen}\left(30^{\circ} *\right.$ $0)+0,150536 \cos \left(60^{\circ} * 0\right)+0,522108 \operatorname{sen}\left(60^{\circ} *\right.$ 


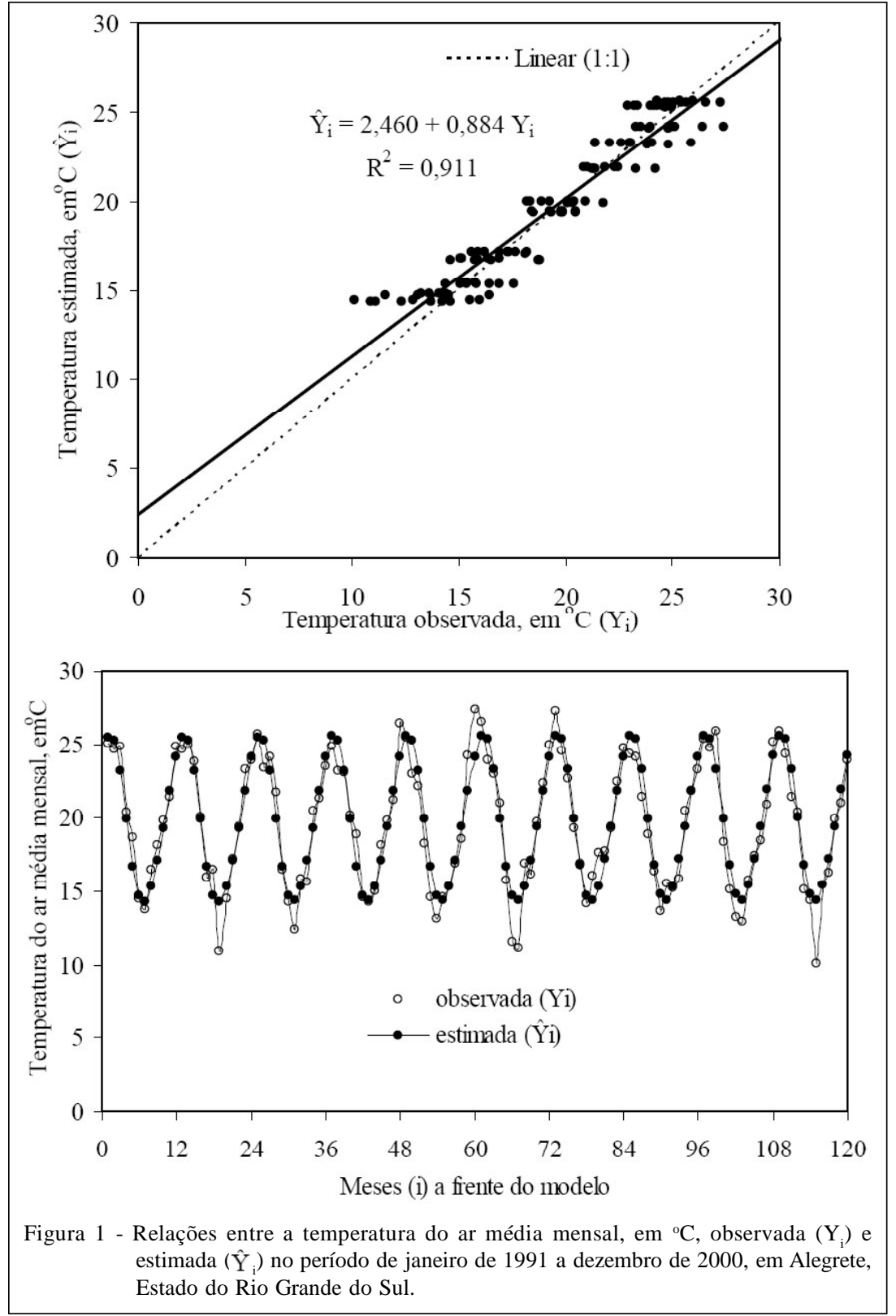

$0)+0,00086263(720+1)=25,5151^{\circ} \mathrm{C}$. Ainda, para o mês de dezembro de 2000, em Alegrete, $\hat{\mathrm{Y}}_{120}=19,135828+5,606824 \cos \left(30^{\circ} * 11\right)+0,285129 \mathrm{sen}$ $\left(30^{\circ} * 11\right)+0,150536 \cos \left(60^{\circ} * 11\right)+0,522108 \operatorname{sen}\left(60^{\circ} *\right.$ $11)+0,00086263(720+120)=24,1966^{\circ}$ C. Do ponto de vista prático, no futuro, a partir de novos dados observados, é importante que as estimativas dos parâmetros do modelo (Tabela 3) sejam ajustadas para incorporar possíveis mudanças de tendências nas séries temporais. Além da previsão de valores futuros, esses modelos são adequados para estimar falha de dados.

\section{CONCLUSÃO}

O modelo harmônico gerado, para cada um dos 37 locais do Estado do Rio Grande do Sul, é adequado para estimar a temperatura do ar média mensal.

\section{AGRADECIMENTOS}

Aos pesquisadores, aos técnicos, aos observadores meteorológicos, aos estagiários e às demais pessoas que de alguma forma contribuíram para a realização deste trabalho; e ao Conselho Nacional de Desenvolvimento Científico e 
Tecnológico (CNPq), pela concessão de bolsa de produtividade em pesquisa para Alberto Cargnelutti Filho.

\section{REFERÊNCIAS}

AMARAl, E. Análise harmônica. Pesquisa Agropecuária Brasileira, v.3, p.7-43, 1968.

ASSIS, F.N. de et al. Aplicações de estatística à climatologia: teoria e prática. Pelotas: UFPEL, 1996. 161p.

CAMARgO, A.P.de; SENTElHAS, P.C. Avaliação do desempenho de diferentes métodos de estimativa da evapotranspiração potencial no estado de São Paulo, Brasil. Revista Brasileira de Agrometeorologia, v.5, p.89-97, 1997.

CARGNELUTTI FILHO, A. et al. Ajustes de funções de distribuição de probabilidade à temperatura máxima do ar no Rio Grande do Sul. Pesquisa Agropecuária Gaúcha, v.14, p.87-93, 2008a.

CARGNELUTTI FILHO, A. et al. Altitude e coordenadas geográficas na estimativa da temperatura mínima média decendial do ar no estado do Rio Grande do Sul. Pesquisa Agropecuária Brasileira, v.41, p.893-901, 2006. Disponível em: <http:// www.scielo.br/scielo.php?script $=$ sci_arttext\&pid $=$ S0100204X2006000600001\&lng=pt\&nrm=iso>. Acesso em: $12 \mathrm{fev}$. 2010. doi: $10.1590 / \mathrm{S} 0100-204 \mathrm{X} 2006000600001$.

CARGNELUTTI FILHO, A. et al. Coordenadas geográficas na estimativa das temperaturas máxima e média decendiais do ar no Estado do Rio Grande do Sul. Ciência Rural, v.38, p.24482456, 2008b. Disponível em: <http://www.scielo.br/ scielo.php ? script $=$ sci_arttext \& pid = S $0103-$ $84782008000900006 \& \operatorname{lng}=\mathrm{pt} \& \mathrm{nrm}=\mathrm{iso}>$. Acesso em: $12 \mathrm{fev}$. 2010. doi: 10.1590/S0103-84782008005000005.
JOÃO, M.M.L. Análise do comportamento das temperaturas máxima e mínima médias mensais para o Estado do Rio Grande do Sul. 2009. 137f. Dissertação (Mestrado em Meteorologia) - Programa de Pós-graduação em Meteorologia, Universidade Federal de Pelotas. Disponível em: <http:// www.ufpel.tche.br/meteorologia/pos-graduacao/dissertacoes/ dissertacoes_completas/dissertacao_milena_joao.pdf $>$. Acesso em: 12 fev. 2010

MEZZOMO, D. et al. Análise espectral da temperatura mínima média em dois trimestres numa região homogênea do Rio Grande do Sul. Revista Brasileira de Agrometeorologia, v.15, p.121-132, 2007. Disponível em: <http://www.sbagro.org.br/ rbagro/ojs/index.php/rbagro/article/viewFile/57/1>. Acesso em: 12 fev. 2010.

MORETTIN, P.A.; TOLOI, C.M.C. Análise de séries temporais. São Paulo: Edgard Blücher, 2006. 564p.

SIEGEL, S.; CASTELLAN JÚNIOR, N.J. Estatística nãoparamétrica para ciências do comportamento. 2.ed. Porto Alegre: Artmed, 2006. 448p.

SILVA, J.B. da; FERNANDES, A. M. Análise harmônica das temperaturas mínimas em Pelotas, RS. Revista Brasileira de Meteorologia, v.6, p.455-470, 1991.

SARTORIS, A. Estatística e introdução à econometria. São Paulo: Saraiva, 2003. 426p.

STEEL, R.G.D. et al. Principles and procedures of statistics a biometrical approach. 3.ed. Nova York: McGraw-Hill, 1997. 666p.

WILlmotT, C.J. On the validation of models. Physical Geography, v.2, p.184-194, 1981. 